\title{
Inversion Formulas for the Spherical Radon-Dunkl Transform ${ }^{\star}$
}

\author{
Zhongkai LI and Futao SONG
}

Department of Mathematics, Capital Normal University, Beijing 100048, China

E-mail: lizk@mail.cnu.edu.cn,ftsong@tom.com

Received October 18, 2008, in final form March 01, 2009; Published online March 03, 2009

doi:10.3842/SIGMA.2009.025

\begin{abstract}
The spherical Radon-Dunkl transform $R_{\kappa}$, associated to weight functions invariant under a finite reflection group, is introduced, and some elementary properties are obtained in terms of $h$-harmonics. Several inversion formulas of $R_{\kappa}$ are given with the aid of spherical Riesz-Dunkl potentials, the Dunkl operators, and some appropriate wavelet transforms.
\end{abstract}

Key words: spherical Radon-Dunkl transform; $h$-harmonics; inversion formula; wavelet

2000 Mathematics Subject Classification: 44A12; 33C55; 65R32; 42C40

\section{Introduction}

Let $\langle x, y\rangle$ denote the usual Euclidean inner product of $x, y \in \mathbb{R}^{d+1}$, and $\mathbb{S}^{d}=\{x:\|x\|=1\}$ the unit sphere in $\mathbb{R}^{d+1}$. We use $d \omega_{k}$ to denote the surface (Lebesgue) measure on a $k$-dimensional sphere. The spherical Radon transform $R$ is one of the tools in integral geometry, which is defined, for $f \in C\left(\mathbb{S}^{d}\right)$, by

$$
R f(x)=\Lambda_{d-1} \int_{\langle x, y\rangle=0} f(y) d \omega_{d-1}(y), \quad x \in \mathbb{S}^{d},
$$

where $\Lambda_{d-1}^{-1}$ is the surface area of $\mathbb{S}^{d-1}$. There are a number of papers devoting to the study of the spherical Radon transform $R$ by different methods (see $[1,13,14,15,16,17,21,22,23,24$, $25,26,27,28]$ ), and to its applications to various problems (see [10, 11]). Some deep results about $R$ were obtained with the aid of spherical harmonics (see [21, 22, 23, 24, 25, 26, 27, 28]), and furthermore, $R$ is a special case of the spherical means

$$
M_{\tau} f(x)=\frac{\Lambda_{d-1}}{\left(1-\tau^{2}\right)^{(d-1) / 2}} \int_{\langle x, y\rangle=\tau} f(y) d \omega_{d-1}(y), \quad x \in \mathbb{S}^{d},
$$

by taking $\tau=0$, and also of the spherical Riesz potentials

$$
I^{\alpha} f(x)=\frac{\Gamma((1-\alpha) / 2)}{2 \pi^{d / 2} \Gamma(\alpha / 2)} \int_{\mathbb{S}^{d}}|\langle x, y\rangle|^{\alpha-1} f(y) d \omega_{d}(y), \quad x \in \mathbb{S}^{d}
$$

by taking the limit $\lim _{\alpha \rightarrow 0+} I^{\alpha} f=R f$ in some sense (see [22]). The former is a tool in approximation on the sphere $\mathbb{S}^{d}$, and the later is one of the research objectives in harmonic analysis on $\mathbb{S}^{d}$.

The purpose of the present paper is to study an analogous model of the spherical Radon transform $R$ in Dunkl's theory. This is based on the definition of the generalized spherical

\footnotetext{
${ }^{\star}$ This paper is a contribution to the Special Issue on Dunkl Operators and Related Topics. The full collection is available at http://www.emis.de/journals/SIGMA/Dunkl_operators.html
} 
means $M_{\tau}^{\kappa} f(x)$ due to [33] (instead of $M_{\tau}^{\kappa}$, the notation $T_{\theta}^{\kappa}$ with $\tau=\cos \theta$ was used there), in terms of the equation

$$
\int_{-1}^{1} M_{\tau}^{\kappa} f(x) g(\tau) w_{\lambda_{\kappa}}(\tau) d \tau=c_{\kappa} \int_{\mathbb{S}^{d}} f(y) V_{\kappa}[g(\langle x, \cdot\rangle)](y) h_{\kappa}^{2}(y) d \omega_{d}(y)
$$

for any $g$ in $L^{1}\left([-1,1] ; w_{\lambda_{\kappa}}\right)$, where $w_{\lambda_{\kappa}}(t)=\tilde{c}_{\lambda_{\kappa}+1 / 2}\left(1-t^{2}\right)^{\lambda_{\kappa}-1 / 2}, V_{\kappa}$ is the intertwining operator associated to a given finite reflection group, and $h_{\kappa}^{2}$ is the related weight function (for details concerning them and other notations in the equation, see the next section). We define $R_{\kappa}$ by $R_{\kappa} f=M_{0}^{\kappa} f$, and call $R_{\kappa}$ the spherical Radon-Dunkl transform. Although $M_{\tau}^{\kappa}$ is defined implicitly, it is a proper extension of $M_{\tau}$ and $M_{\tau}^{0}=M_{\tau}$, and moreover, from [2] and [33, 34, 35, 36], $M_{\tau}^{\kappa}$ shares many properties with $M_{\tau}$ and plays the same roles in weighted approximation and related harmonic analysis on the sphere $\mathbb{S}^{d}$. One could expect that the spherical Radon-Dunkl Transform $R_{\kappa}$ would have similar features to $R$ and be a suitable tool in reconstruction of functions in weighted spaces. This is the motivation of the paper. Despite less closed representation, a further work worth doing is to find applications of $R_{\kappa}$ in geometry or other fields.

The paper is organized as follows. In Section 2, some necessary facts in Dunkl's theory are reviewed, and in Section 3, the spherical Radon-Dunkl transform $R_{\kappa}$ is defined and some of elementary properties are obtained in terms of $h$-harmonics. Sections 4 and 5 are devoted to inversion formulas of $R_{\kappa}$, which are given by means of spherical Riesz-Dunkl potentials $I_{\kappa}^{\alpha}$, the Dunkl operators, and some appropriate wavelet transforms. These conclusions generalize part of those in $[21,22,23]$.

\section{Some facts in Dunkl's theory}

Let $G$ be a finite reflection group on $\mathbb{R}^{d+1}$ with a fixed positive root system $R_{+}$, normalized so that $\langle v, v\rangle=2$ for all $v \in R_{+}$. It is known that $G$ is a subgroup of $O(d+1)$ generated by $\left\{\sigma_{v}\right.$ : $\left.v \in R_{+}\right\}$, where $\sigma_{v}$ denotes the reflection with respect to the hyperplane perpendicular to $v$, i.e. $x \sigma_{v}=x-2(\langle x, v\rangle /\langle v, v\rangle) v$ for $x \in \mathbb{R}^{d+1}$. Let $\kappa$ be a multiplicity function $v \mapsto \kappa_{v} \in[0,+\infty)$ defined on $R_{+}$, with invariance under the action of $G$. Thus $\left\{\kappa_{v}: v \in R_{+}\right\}$has different values only as many as the number of $G$-orbits in $R_{+}$.

The Dunkl operators are a family of first-order differential-reflection operators $\mathcal{D}_{j}, 1 \leq j \leq$ $d+1$, defined by (see [5])

$$
\mathcal{D}_{j} f(x):=\partial_{j} f(x)+\sum_{v \in R_{+}} \kappa_{v} \frac{f(x)-f\left(x \sigma_{v}\right)}{\langle x, v\rangle}\left\langle v, e_{j}\right\rangle,
$$

for $f \in C^{1}\left(\mathbb{R}^{d+1}\right)$, where $\left\{e_{i}: 1 \leq i \leq d+1\right\}$ is the usual standard basis of $\mathbb{R}^{d+1}$. As substitutes of partial differentiations $\partial_{j}$, these operators are mutually commutative. The associated Laplacian, called $h$-Laplacian, is defined by $\Delta_{h}=\mathcal{D}_{1}^{2}+\cdots+\mathcal{D}_{d+1}^{2}$, which plays roles similar to that of the usual Laplacian $\Delta=\Delta_{0}$ (see [4]). In terms of the polarspherical coordinates $x=r x^{\prime}, r=\|x\|$, the operator $\Delta_{h}$ can be expressed as (see [33])

$$
\Delta_{h}=\frac{\partial^{2}}{\partial r^{2}}+\frac{2 \lambda_{\kappa}+1}{r} \frac{\partial}{\partial r}+\frac{1}{r^{2}} \Delta_{h, 0},
$$

where $\Delta_{h, 0}$ is the associated Laplace-Beltrami operator on $\mathbb{S}^{d}$, and $\lambda_{\kappa}=\gamma_{\kappa}+(d-1) / 2$ with $\gamma_{\kappa}=\sum_{v \in R_{+}} \kappa_{v}$. If $\gamma_{\kappa}=0$, i.e. $\kappa_{v} \equiv 0$, then $\mathcal{D}_{j}=\partial_{j}, 1 \leq j \leq d+1$. In the following, we assume that $\gamma_{\kappa}>0$, and so $\lambda_{\kappa}>0$.

For each multiplicity function $\kappa$, there is a linear operator $V_{\kappa}$ intertwining the partial differentiations and the Dunkl operators (see [6]). Precisely, if $\mathcal{P}_{n}=\mathcal{P}_{n}^{d+1}$ denotes the set of homogeneous polynomials of degree $n$ in $d+1$ variables, then the intertwining operator $V_{\kappa}$ is determined 
uniquely by $V_{\kappa} \mathcal{P}_{n} \subseteq \mathcal{P}_{n}, V_{\kappa} 1=1$ and $\mathcal{D}_{j} V_{\kappa}=V_{\kappa} \partial_{j}, 1 \leq i \leq d+1$. $V_{\kappa}$ commutes with the group action and is a linear isomorphism on each $\mathcal{P}_{n}(n=0,1, \ldots)$. Moreover it is a positive operator (see [18]) and can be extended to the space of smooth functions and even to the space of distributions (see $[29,30]$ ).

The intertwining operator $V_{\kappa}$ allows to introduce some useful tools in Dunkl's theory. For example, the Dunkl transform $\mathcal{F}_{\kappa}$ is defined in [7] associated with the measure $h_{\kappa}^{2} d x$ on $\mathbb{R}^{d+1}$, where

$$
h_{\kappa}(x)=\prod_{v \in R_{+}}|\langle x, v\rangle|^{\kappa_{v}} .
$$

$\mathcal{F}_{\kappa}$ is a generalization of the Fourier transform $\mathcal{F}=\mathcal{F}_{0}$ and enjoys properties similar to those of $\mathcal{F}$ (see $[3,7,19])$.

For $1 \leq p<\infty$, denote by $\|f\|_{\kappa, p}=\left\{c_{\kappa} \int_{\mathbb{S}^{d}}|f|^{p} h_{\kappa}^{2} d \omega_{d}\right\}^{1 / p}$ the norm of $f \in L^{p}\left(\mathbb{S}^{d} ; h_{\kappa}^{2}\right)$, with $c_{\kappa}^{-1}=\int_{\mathbb{S}^{d}} h_{\kappa}^{2} d \omega_{d}$, and by $\|\phi\|_{\lambda_{\kappa}, p}=\left\{\int_{-1}^{1}|\phi|^{p} w_{\lambda_{\kappa}} d t\right\}^{1 / p}$ the norm of $\phi \in L^{p}\left([-1,1], w_{\lambda_{\kappa}}\right)$, where $w_{\lambda_{\kappa}}(t)=\tilde{c}_{\lambda_{\kappa}+1 / 2}\left(1-t^{2}\right)^{\lambda_{\kappa}-1 / 2}, \tilde{c}_{\lambda}=\pi^{-1 / 2} \Gamma(\lambda+1 / 2) / \Gamma(\lambda)$. When $p=\infty,\|f\|_{\infty}=\|f\|_{\kappa, \infty}$ and $\|\phi\|_{\infty}=\|\phi\|_{\kappa, \infty}$ are defined as usual.

The functions in $\mathcal{H}_{n}^{h, d+1}:=\mathcal{P}_{n}^{d+1} \cap$ ker $\Delta_{h}$ are called $h$-harmonic polynomials of degree $n$, and the spherical $h$-harmonics of degree $n$ are their restrictions on $\mathbb{S}^{d}$. The orthogonality theorem in [4] asserts that if $P \in \mathcal{P}_{n}^{d+1}$, then $\int_{\mathbb{S}^{d}} P Q h_{\kappa}^{2} d \omega_{d}=0$ for all $Q \in \cup_{k=0}^{n-1} \mathcal{P}_{k}^{d+1}$, if and only if $P$ is $h$-harmonic, i.e. $\Delta_{h} P=0$. Moreover $L^{2}\left(\mathbb{S}^{d} ; h_{\kappa}^{2}\right)=\sum_{n=0}^{\infty} \bigoplus \mathcal{H}_{n}^{h, d+1}$.

If $Y_{n}\left(h_{\kappa}^{2} ; f ; x\right)$ is the projection of $f \in L^{1}\left(\mathbb{S}^{d} ; h_{\kappa}^{2}\right)$ to $\mathcal{H}_{n}^{h, d+1}$, then the $h$-harmonic expansion of $f$ is given by

$$
f(x) \sim \sum_{n=0}^{\infty} Y_{n}\left(h_{\kappa}^{2} ; f ; x\right), \quad x \in \mathbb{S}^{d} .
$$

The projection $Y_{n}\left(h_{\kappa}^{2} ; f ; x\right)$ takes the form

$$
Y_{n}\left(h_{\kappa}^{2} ; f ; x\right)=c_{\kappa} \int_{\mathbb{S}^{d}} f(y) P_{n}\left(h_{\kappa}^{2} ; x, y\right) h_{\kappa}^{2}(y) d \omega_{d}(y),
$$

where $P_{n}\left(h_{\kappa}^{2} ; x, y\right)$ is the reproducing kernel of the space $\mathcal{H}_{n}^{h, d+1}$. A compact formula of $P_{n}\left(h_{\kappa}^{2} ; x, y\right)$ is (see $[32])$

$$
P_{n}\left(h_{\kappa}^{2} ; x, y\right)=\frac{n+\lambda_{\kappa}}{\lambda_{\kappa}} V_{\kappa}\left[C_{n}^{\lambda_{\kappa}}(\langle x, \cdot\rangle)\right](y),
$$

with $C_{n}^{\lambda_{\kappa}}$, the Gegenbauer polynomial of degree $n$ with parameter $\lambda_{\kappa}$. It is noted that (see [33])

$$
\Delta_{h, 0} Y_{n}=-n\left(n+2 \lambda_{\kappa}\right) Y_{n}, \quad Y_{n} \in \mathcal{H}_{n}^{h, d+1} .
$$

When $\kappa_{v}=0$ for all $v \in R_{+}$, we have $V_{0}=i d$, and hence, $P_{n}\left(h_{\kappa}^{2} ; x, y\right)$ reduces to the usual zonal polynomial for the ordinary spherical harmonics $P_{n}(x, y)=\frac{n+(d-1) / 2}{(d-1) / 2} C_{n}^{(d-1) / 2}(\langle x, y\rangle)$.

A useful integration formula for the intertwining operator $V_{\kappa}$ is

$$
\int_{\mathbb{S}^{d}} V_{\kappa} f(x) h_{\kappa}^{2}(x) d \omega_{d}(x)=\frac{c_{\kappa}^{-1} \Gamma\left(\lambda_{\kappa}+1\right)}{\pi^{(d+1) / 2} \Gamma\left(\gamma_{\kappa}\right)} \int_{\mathbb{B}^{d+1}} f(x)\left(1-|x|^{2}\right)^{\gamma_{\kappa}-1} d x .
$$

The formula is proved in [31] when $f$ is a polynomial. Applying density of polynomials and positivity of $V_{\kappa}$, this allows us to extend the intertwining operator $V_{\kappa}$ acting on those functions $f$ 
on the sphere $\mathbb{S}^{d}$ which are restrictions of functions in $L^{1}\left(\mathbb{B}^{d+1} ;\left(1-|x|^{2}\right)^{\gamma_{\kappa}-1}\right)$, and moreover, the formula (5) is true for these functions too and $V_{\kappa} f \in L^{1}\left(\mathbb{S}^{d} ; h_{\kappa}^{2}\right)$. In particular, if $\phi \in$ $L^{1}\left([-1,1] ; w_{\lambda_{\kappa}}\right)$, then for each $y \in \mathbb{S}^{d}$,

$$
\int_{\mathbb{B}^{d+1}} \phi(\langle x, y\rangle)\left(1-|x|^{2}\right)^{\gamma_{\kappa}-1} d x=\frac{\pi^{(d+1) / 2} \Gamma\left(\gamma_{\kappa}\right)}{\Gamma\left(\lambda_{\kappa}+1\right)} \int_{-1}^{1} \phi(t) w_{\lambda_{\kappa}}(t) d t,
$$

i.e. $f(x)=\phi(\langle x, y\rangle) \in L^{1}\left(\mathbb{B}^{d+1} ;\left(1-|x|^{2}\right)^{\gamma_{\kappa}-1}\right)$, and hence $V_{\kappa}[\phi(\langle\cdot, y\rangle)]$ is well defined and in $L^{1}\left(\mathbb{S}^{d} ; h_{\kappa}^{2}\right)$. In addition, we have the following symmetric relation

$$
V_{\kappa}[\phi(\langle\cdot, y\rangle)](x)=V_{\kappa}[\phi(\langle x, \cdot\rangle)](y), \quad \text { for a.e. } \quad(x, y) \in \mathbb{S}^{d} \times \mathbb{S}^{d} .
$$

The validity of $(7)$ for polynomials and for all $(x, y) \in \mathbb{S}^{d} \times \mathbb{S}^{d}$ follows from Gegenbauer expansions and the symmetry of the reproducing kernels $P_{n}\left(h_{\kappa}^{2} ; x, y\right)$, If $\phi \in L^{1}\left([-1,1] ; w_{\lambda_{\kappa}}\right)$ and $\phi_{1}$ is a univariate polynomial, then applying (5) and (6),

$$
\begin{aligned}
\int_{\mathbb{S}^{d}} \int_{\mathbb{S}^{d}}\left|V_{\kappa}[\phi(\langle\cdot, y\rangle)](x)-V_{\kappa}[\phi(\langle x, \cdot\rangle)](y)\right| h_{\kappa}^{2}(x) h_{\kappa}^{2}(y) d \omega_{d}(x) d \omega_{d}(y) \\
\quad=\int_{\mathbb{S}^{d}} \int_{\mathbb{S}^{d}}\left|V_{\kappa}\left[\left(\phi-\phi_{1}\right)(\langle\cdot, y\rangle)\right](x)-V_{\kappa}\left[\left(\phi-\phi_{1}\right)(\langle x, \cdot\rangle)\right](y)\right| h_{\kappa}^{2}(x) h_{\kappa}^{2}(y) d \omega_{d}(x) d \omega_{d}(y) \\
\quad \leq 2 c_{\kappa}^{-2} \int_{-1}^{1}\left|\phi(t)-\phi_{1}(t)\right| w_{\lambda_{\kappa}}(t) d t,
\end{aligned}
$$

which implies $(7)$ by the density of polynomials in $L^{1}\left([-1,1] ; w_{\lambda_{\kappa}}\right)$. Following the above remarks, the Funk-Hecke formula for $h$-harmonics proved in [32] (for continuous functions there only) holds also for $\phi \in L^{1}\left([-1,1] ; w_{\lambda_{\kappa}}\right)$, that is

$$
c_{\kappa} \int_{\mathbb{S}^{d}} V_{\kappa}[\phi(\langle\cdot, y\rangle)](x) H_{n}(x) h_{\kappa}^{2}(x) d \omega_{d}(x)=L_{n}(\phi) H_{n}(y)
$$

for each $H_{n} \in \mathcal{H}_{n}^{h, d+1}$ and $y \in \mathbb{S}^{d}$, where

$$
L_{n}(\phi)=\int_{-1}^{1} \phi(t) \frac{C_{n}^{\lambda_{\kappa}}(t)}{C_{n}^{\lambda_{\kappa}}(1)} w_{\lambda_{\kappa}}(t) d t
$$

The convolution $f *_{\kappa} \phi$ of two functions $f \in L^{1}\left(\mathbb{S}^{d} ; h_{\kappa}^{2}\right)$ and $\phi \in L^{1}\left([-1,1] ; w_{\lambda_{\kappa}}\right)$ is defined in [34], by

$$
f *_{\kappa} \phi(x)=c_{\kappa} \int_{\mathbb{S}^{d}} f(y) V_{\kappa}[\phi(\langle x, \cdot\rangle)](y) h_{\kappa}^{2}(y) d \omega_{d}(y) .
$$

The Young inequality concerning such convolution is proved in [34], that is, for $p, q, r \geq 1$ with $r^{-1}=p^{-1}+q^{-1}-1$,

$$
\left\|f *_{\kappa} \phi\right\|_{\kappa, r} \leq\|f\|_{\kappa, p}\|\phi\|_{\lambda_{\kappa}, q} .
$$

A typical example of Dunkl's theory is the case when $G=Z_{2}^{d+1}$, for which, the function $h_{\kappa}(x)$ has the form $h_{\kappa}(x)=\left|x_{1}\right|^{\kappa_{1}} \cdots\left|x_{d+1}\right|^{\kappa_{d+1}}$ and the intertwining operator $V_{\kappa}$ is given by

$$
V_{\kappa} f(x)=\tilde{c}_{\kappa} \int_{[-1,1]^{d+1}} f\left(x_{1} t_{1}, \ldots, x_{d+1} t_{d+1}\right) \prod_{i=1}^{d+1}\left(1+t_{i}\right)\left(1-t_{i}^{2}\right)^{\kappa_{i}-1} d t_{1} \cdots d t_{d+1},
$$

where $\tilde{c}_{\kappa}=\tilde{c}_{\kappa_{1}} \cdots \tilde{c}_{\kappa_{d+1}}$. 


\section{The spherical Radon-Dunkl transform}

For $f \in L^{1}\left(\mathbb{S}^{d} ; h_{\kappa}^{2}\right)$, its generalized spherical means $M_{\tau}^{\kappa} f(x)$ due to [33] is defined by the equation

$$
\int_{-1}^{1} M_{\tau}^{\kappa} f(x) \phi(\tau) w_{\lambda_{\kappa}}(\tau) d \tau=f *_{\kappa} \phi(x)
$$

for any $\phi$ in $L^{1}\left([-1,1] ; w_{\lambda_{\kappa}}\right)$. Since, for $\phi \in L^{\infty}\left(\mathbb{S}^{d}\right)$,

$$
\left|\int_{-1}^{1} M_{\tau}^{\kappa} f(x) \phi(\tau) w_{\lambda_{\kappa}}(\tau) d \tau\right| \leq\|f\|_{\kappa, 1}\|\phi\|_{\infty}
$$

it follows that, for each $x \in \mathbb{S}^{d}$, the function $\psi_{x}(\tau)=M_{\tau}^{\kappa} f(x) \in L^{1}\left([-1,1] ; w_{\lambda_{\kappa}}\right)$. This shows that for almost all $\tau \in[-1,1], M_{\tau}^{\kappa} f$ is well defined. To give further illustration of $M_{\tau}^{\kappa}$, we introduce the space $W_{m}^{p}\left(\mathbb{S}^{d} ; h_{\kappa}^{2}\right)\left(\subseteq L^{p}\left(\mathbb{S}^{d} ; h_{\kappa}^{2}\right)\right)$ of functions for $m \geq 0$, such that for $f \in W_{m}^{p}\left(\mathbb{S}^{d} ; h_{\kappa}^{2}\right)$, there exist some $g \in L^{p}\left(\mathbb{S}^{d} ; h_{\kappa}^{2}\right)$ satisfying $Y_{0}\left(h_{\kappa}^{2} ; f\right)=Y_{0}\left(h_{\kappa}^{2} ; g\right)$ and $\left[n\left(n+2 \lambda_{\kappa}\right)\right]^{m / 2} Y_{n}\left(h_{\kappa}^{2} ; f\right)=$ $Y_{n}\left(h_{\kappa}^{2} ; g\right)$ for all $n=1,2, \ldots$ In view of $(4)$, we formally write $g=\left(-\Delta_{h, 0}\right)^{m / 2} f$. It is noted that for even $m, C^{m}\left(\mathbb{S}^{d}\right) \subseteq W_{m}^{p}\left(\mathbb{S}^{d} ; h_{\kappa}^{2}\right)(1 \leq p \leq \infty)$. The following properties of $M_{\tau}^{\kappa}$ are proved in $[33,34]$.

\section{Proposition 1.}

(i) If $f_{0}(x) \equiv 1$, then $M_{\tau}^{\kappa} f_{0}(x) \equiv 1$.

(ii) For each $\tau \in[-1,1]$, there is an extension of $M_{\tau}^{\kappa}$ to $L^{p}\left(\mathbb{S}^{d} ; h_{\kappa}^{2}\right)(1 \leq p<\infty)$, or $C\left(\mathbb{S}^{d}\right)$ $(p=\infty)$, such that

$$
\left\|M_{\tau}^{\kappa} f\right\|_{\kappa, p} \leq\|f\|_{\kappa, p}, \quad \tau \in[-1,1]
$$

(iii) For $f \in L^{1}\left(\mathbb{S}^{d} ; h_{\kappa}^{2}\right)$,

$$
Y_{n}\left(h_{\kappa}^{2} ; M_{\tau}^{\kappa} f ; x\right)=\frac{C_{n}^{\lambda_{\kappa}}(\tau)}{C_{n}^{\lambda_{\kappa}}(1)} Y_{n}\left(h_{\kappa}^{2} ; f ; x\right),
$$

and in particular, $\Delta_{h, 0}\left(M_{\tau}^{\kappa} f\right)=M_{\tau}^{\kappa}\left(\Delta_{h, 0} f\right)$ if $\Delta_{h, 0} f \in L^{1}\left(\mathbb{S}^{d} ; h_{\kappa}^{2}\right)$.

Proof. Here we give an independent, but simpler proof for part (ii) as follows. For all $\phi \in$ $L^{1}\left([-1,1] ; w_{\lambda_{\kappa}}\right)$ and $g \in L^{p^{\prime}}\left(\mathbb{S}^{d} ; h_{\kappa}^{2}\right)$, it follows from (13) that

$$
\int_{-1}^{1} \phi(\tau) \xi(\tau) w_{\lambda_{\kappa}}(\tau) d \tau=c_{\kappa} \int_{\mathbb{S}^{d}}\left(f *_{\kappa} \phi\right)(x) g(x) h_{\kappa}^{2}(x) d \omega_{d}(x)
$$

where $\xi(\tau)=c_{\kappa} \int_{\mathbb{S}^{d}} M_{\tau}^{\kappa} f(x) \cdot g(x) h_{\kappa}^{2}(x) d \omega_{d}(x)$. By using (7) and (10), the right-hand side above becomes $c_{\kappa} \int_{\mathbb{S}^{d}} f(y)\left(g *_{\kappa} \phi\right)(y) h_{\kappa}^{2}(y) d \omega_{d}(y)$, and then, by applying Hölder's inequality and the Young inequality (11), its absolute value is dominated by $\|f\|_{\kappa, p}\|g\|_{\kappa, p^{\prime}}\|\phi\|_{\lambda_{\kappa}, 1}$. This gives that $\sup _{\tau \in[-1,1]}|\xi(\tau)| \leq\|f\|_{\kappa, p}\|g\|_{\kappa, p^{\prime}}$, which means that, for almost all $\tau \in[-1,1],\left\|M_{\tau}^{\kappa} f\right\|_{\kappa, p} \leq$ $\|f\|_{\kappa, p}$. If $f$ is a polynomial, part (iii) implies that $M_{\tau}^{\kappa} f(x)$ is a continuous function of $(\tau, x) \in$ $[-1,1] \times \mathbb{S}^{d}$, so that $\left\|M_{\tau}^{\kappa} f\right\|_{\kappa, p} \leq\|f\|_{\kappa, p}$ is true for all $\tau \in[-1,1]$ in this case. Finally, from density of the set of polynomials, for each $\tau \in[-1,1], M_{\tau}^{\kappa}$ can be extended to all functions in $L^{p}\left(\mathbb{S}^{d} ; h_{\kappa}^{2}\right)(1 \leq p<\infty)$, or $C\left(\mathbb{S}^{d}\right)$. Following this, part $(i i i)$ also holds for $f \in L^{1}\left(\mathbb{S}^{d} ; h_{\kappa}^{2}\right)$ and each $\tau \in[-1,1]$, and moreover,

$$
M_{\tau}^{\kappa} f \sim \sum_{n=0}^{\infty} \frac{C_{n}^{\lambda_{\kappa}}(\tau)}{C_{n}^{\lambda_{\kappa}}(1)} Y_{n}\left(h_{\kappa}^{2} ; f ; x\right) .
$$

We note that when $f$ is even in $\mathbb{S}^{d}, M_{\tau} f$ is even for $\tau \in(-1,1)$. 
The following proposition gives a pointwise description of $M_{\tau}^{\kappa}$ for a larger class of functions.

Proposition 2. For $f \in W_{m}^{2}\left(\mathbb{S}^{d} ; h_{\kappa}^{2}\right)$ with $m>\lambda_{\kappa}+1, M_{\tau}^{\kappa} f(x)$ is a continuous function of $(\tau, x) \in[-1,1] \times \mathbb{S}^{d}$ and

$$
M_{\tau}^{\kappa} f(x)=\sum_{n=0}^{\infty} \frac{C_{n}^{\lambda_{\kappa}}(\tau)}{C_{n}^{\lambda_{\kappa}}(1)} Y_{n}\left(h_{\kappa}^{2} ; f ; x\right),
$$

the series on the right-hand side being absolutely and uniformly convergent.

Proof. It is noted that for $t \in[-1,1],\left|C_{n}^{\lambda_{\kappa}}(t)\right| \leq C_{n}^{\lambda_{\kappa}}(1)=\left(2 \lambda_{\kappa}\right)_{n} / n ! \simeq n^{2 \lambda_{\kappa}-1}$ [8, p. 19]. From (2), for $g \in L^{2}\left(\mathbb{S}^{d} ; h_{\kappa}^{2}\right)$ and all $x \in \mathbb{S}^{d}$ we have $\left|Y_{n}\left(h_{\kappa}^{2} ; g ; x\right)\right| \leq\|g\|_{\kappa, 2}\left\|P_{n}\left(h_{\kappa} ; x, \cdot\right)\right\|_{\kappa, 2}$. In view of orthogonality of $h$-harmonics and from (3),

$$
\left\|P_{n}\left(h_{\kappa} ; x, \cdot\right)\right\|_{\kappa, 2}^{2}=P_{n}\left(h_{\kappa} ; x, x\right) \leq \lambda_{\kappa}^{-1}\left(n+\lambda_{\kappa}\right) C_{n}^{\lambda_{\kappa}}(1) \simeq \lambda_{\kappa}^{-1} n^{2 \lambda_{\kappa}} .
$$

Therefore $\left|Y_{n}\left(h_{\kappa}^{2} ; g ; x\right)\right| \leq c\|g\|_{\kappa, 2} n^{\lambda_{\kappa}}$. If $f \in W_{m}^{2}\left(\mathbb{S}^{d} ; h_{\kappa}^{2}\right)$, then for $n \geq 1, Y_{n}\left(h_{\kappa}^{2} ; f ; x\right)=[n(n+$ $\left.\left.2 \lambda_{\kappa}\right)\right]^{-m / 2} Y_{n}\left(h_{\kappa}^{2} ;\left(-\Delta_{h, 0}\right)^{m / 2} f ; x\right)$, so that $\left|Y_{n}\left(h_{\kappa}^{2} ; f ; x\right)\right| \leq c\left\|\left(-\Delta_{h, 0}\right)^{m / 2} f\right\|_{\kappa, 2} n^{\lambda_{\kappa}-m}$. Hence, when $m>\lambda_{\kappa}+1$, the series in (14) converges absolutely and uniformly for $(\tau, x) \in[-1,1] \times \mathbb{S}^{d}$. In view of the uniqueness of $h$-harmonic expansion following from its Cesàro summability (see [31]), the conclusions in the proposition are proved.

Now we define the transform $R_{\kappa}$ by

$$
R_{\kappa} f=M_{0}^{\kappa} f,
$$

and call $R_{\kappa}$ the spherical Radon-Dunkl transform. By Proposition $1(i i), R_{\kappa} f$ is well defined for $f \in L^{1}\left(\mathbb{S}^{d} ; h_{\kappa}^{2}\right)$, and moreover, from Propositions 1 and 2 , we have the following corollary.

\section{Corollary 1.}

(i) For $f \in L^{p}\left(\mathbb{S}^{d} ; h_{\kappa}^{2}\right)(1 \leq p<\infty)$, or $f \in C\left(\mathbb{S}^{d}\right)(p=\infty)$, we have $\left\|R_{\kappa} f\right\|_{\kappa, p} \leq\|f\|_{\kappa, p}$, and

$$
R_{\kappa} f \sim \sum_{n=0}^{\infty} b_{n} Y_{n}\left(h_{\kappa}^{2} ; f ; x\right),
$$

where

$$
b_{n}= \begin{cases}(-1)^{\frac{n}{2}} \frac{\Gamma\left(\lambda_{\kappa}+1 / 2\right)}{\Gamma(1 / 2)} \frac{\Gamma((n+1) / 2)}{\Gamma\left(\lambda_{\kappa}+(n+1) / 2\right)}, & \text { for } n \text { even } \\ 0, & \text { for } n \text { odd } .\end{cases}
$$

(ii) For $f \in W_{m}^{2}\left(\mathbb{S}^{d} ; h_{\kappa}^{2}\right)$ with $m>\lambda_{\kappa}+1, R_{\kappa} f(x)$ is a continuous function on $\mathbb{S}^{d}$ and

$$
R_{\kappa} f(x)=\sum_{n=0}^{\infty} b_{n} Y_{n}\left(h_{\kappa}^{2} ; f ; x\right),
$$

where the series on the right-hand side is absolutely and uniformly convergent.

The numbers $b_{n}=C_{n}^{\lambda_{\kappa}}(0) / C_{n}^{\lambda_{\kappa}}(1)$ are computed by using 10-9(3) and 10-9(19) in [9].

The following is a nontrivial example of $R_{\kappa}$. We consider the group $G=Z_{2}^{d+1}$, with $\kappa=$ $\left(\kappa_{1}, 0, \ldots, 0\right)$ and $\kappa_{1}>0$. In this case, $h_{\kappa}(x)=\left|x_{1}\right|^{\kappa_{1}}$ and the intertwining operator $V_{\kappa}$ in (12) reduces to

$$
V_{\kappa} f(x)=\tilde{c}_{\kappa_{1}} \int_{-1}^{1} f\left(x_{1} t, \tilde{x}\right)(1+t)\left(1-t^{2}\right)^{\kappa_{1}-1} d t,
$$

where $x=\left(x_{1}, \tilde{x}\right)$ with $\tilde{x}=\left(x_{2}, \ldots, x_{d+1}\right) \in \mathbb{R}^{d}$. 
We shall show that, for $f \in C\left(\mathbb{S}^{d}\right)$ and for $x_{1} \neq 0$,

$$
M_{\tau}^{\kappa} f(x)=\frac{c_{\kappa} \tilde{c}_{\kappa_{1}} w_{\lambda_{\kappa}}^{-1}(\tau)}{\left|x_{1}\right|^{2 \kappa_{1}}} \int_{\Omega_{\tau}} f(y)|\langle x, y\rangle-\tau|^{\kappa_{1}-1}|\langle x \sigma, y\rangle-\tau|^{\kappa_{1}} d \omega_{d}(y)
$$

where $\Omega_{\tau}=\left\{y \in \mathbb{S}^{d}: y=\left(y_{1}, \tilde{y}\right)\right.$ with $\left.|\langle\tilde{x}, \tilde{y}\rangle-\tau|<\left|x_{1} y_{1}\right|\right\}$, and $\sigma$ is the reflection such that $x \sigma=\left(-x_{1}, x_{2}, \ldots, x_{d+1}\right)$. Indeed, from the above formula for $V_{\kappa}$,

$$
V_{\kappa}[\phi(\langle x, \cdot\rangle)](y)=\tilde{c}_{\kappa_{1}} \int_{-1}^{1} \phi\left(x_{1} y_{1} t+\langle\tilde{x}, \tilde{y}\rangle\right)(1+t)\left(1-t^{2}\right)^{\kappa_{1}-1} d t .
$$

When $y_{1} \neq 0$, taking the substitution of variables $t=(\tau-\langle\tilde{x}, \tilde{y}\rangle) /\left(x_{1} y_{1}\right)$, we get

$$
V_{\kappa}[\phi(\langle x, \cdot\rangle)](y)=\frac{\tilde{c}_{\kappa_{1}}}{\left|x_{1} y_{1}\right|^{2 \kappa_{1}}} \int_{\langle\tilde{x}, \tilde{y}\rangle-\left|x_{1} y_{1}\right|}^{\langle\tilde{x}, \tilde{y}\rangle+\left|x_{1} y_{1}\right|} \phi(\tau)|\langle x, y\rangle-\tau|^{\kappa_{1}-1}|\langle x \sigma, y\rangle-\tau|^{\kappa_{1}} d \tau .
$$

Substituting this into the definition (10) of $f *_{\kappa} \phi$, we have

$$
f *_{\kappa} \phi(x)=\int_{-1}^{1} A_{\tau} f(x) \phi(\tau) w_{\lambda_{\kappa}}(\tau) d \tau
$$

for all $\phi \in L^{1}\left([-1,1] ; w_{\lambda_{\kappa}}\right)$, where $A_{\tau} f(x)$ denotes the expression on the right-hand side of (17). Then from (13), $A_{\tau} f(x)=M_{\tau}^{\kappa} f(x)$, so that (17) is proved.

Taking $\tau=0$ in (17), we get that, for $f \in C\left(\mathbb{S}^{d}\right)$ and for $x_{1} \neq 0$,

$$
R_{\kappa} f(x)=\frac{c_{\kappa} \tilde{c}_{\kappa_{1}} \tilde{c}_{\lambda_{\kappa}+1 / 2}^{-1}}{\left|x_{1}\right|^{2 \kappa_{1}}} \int_{\Omega_{0}} f(y)|\langle x, y\rangle|^{\kappa_{1}-1}|\langle x \sigma, y\rangle|^{\kappa_{1}} d \omega_{d}(y) .
$$

\section{Inversion formulas for $\boldsymbol{R}_{\kappa}$ by means of spherical Riesz-Dunkl potentials}

For $f \in L^{1}\left(\mathbb{S}^{d} ; h_{\kappa}^{2}\right)$ and $\Re \alpha>0, \alpha \neq 1,3,5, \ldots$, we define its spherical Riesz-Dunkl potential $I_{\kappa}^{\alpha} f$ by

$$
I_{\kappa}^{\alpha} f(x)=C_{\kappa, \alpha} \int_{\mathbb{S}^{d}} f(y) V_{\kappa}\left(|\langle x, \cdot\rangle|^{\alpha-1}\right)(y) h_{\kappa}^{2}(y) d \omega_{d}(y),
$$

where $C_{\kappa, \alpha}=\frac{\sqrt{\pi} \Gamma((1-\alpha) / 2)}{\Gamma\left(\lambda_{\kappa}+1\right) \Gamma(\alpha / 2)} c_{\kappa}$.

Proposition 3. For $\Re \alpha>0, \alpha \neq 1,3,5, \ldots, I_{\kappa}^{\alpha} f$ is well defined for each $f \in L^{1}\left(\mathbb{S}^{d} ; h_{\kappa}^{2}\right)$, and moreover, we have the following statements:

(i) for $1 \leq p \leq \infty$, there exists a constant $c>0$, such that for all $f \in L^{p}\left(\mathbb{S}^{d} ; h_{\kappa}^{2}\right),\left\|I_{\kappa}^{\alpha} f\right\|_{\kappa, p} \leq$ $c\|f\|_{\kappa, p}$

(ii) if the h-harmonic expansion of a function $f \in L^{1}\left(\mathbb{S}^{d} ; h_{\kappa}^{2}\right)$ is given by $(1)$, then $I_{\kappa}^{\alpha} f$ has the following expansion

$$
I_{\kappa}^{\alpha} f(x) \sim \sum_{n=0}^{\infty} b_{n, \alpha} Y_{n}\left(h_{\kappa}^{2} ; f ; x\right), \quad x \in \mathbb{S}^{d},
$$

where

$$
b_{n, \alpha}= \begin{cases}(-1)^{\frac{n}{2}} \frac{\Gamma((n+1-\alpha) / 2)}{\Gamma\left(\lambda_{\kappa}+(n+1+\alpha) / 2\right)}, & \text { for } n \text { even } \\ 0, & \text { for } n \text { odd } .\end{cases}
$$


The conclusions in the proposition are contained in Proposition 2.9 of [34]. Here we give a short presentation. Since $I_{\kappa}^{\alpha} f=c f *_{\kappa} \phi$ with $\phi(t)=|t|^{\alpha-1} \in L^{1}\left([-1,1] ; w_{\lambda_{\kappa}}\right)$, part $(i)$ follows from the Young inequality (11) immediately. For part $(i i)$, since $V_{\kappa}\left(|\langle\cdot, y\rangle|^{\alpha-1}\right) \in L^{1}\left(\mathbb{S}^{d} ; h_{\kappa}^{2}\right)$ from (5) and (6), we have, using (2), (8) and (9),

$$
Y_{n}\left(h_{\kappa}^{2} ; V_{\kappa}\left(|\langle\cdot, y\rangle|^{\alpha-1}\right) ; x\right)=L_{n} P_{n}\left(h_{\kappa}^{2} ; x, y\right),
$$

where

$$
L_{n}=\int_{-1}^{1}|t|^{\alpha-1} \frac{C_{n}^{\lambda_{\kappa}}(t)}{C_{n}^{\lambda_{\kappa}}(1)} w_{\lambda_{\kappa}}(t) d t
$$

From (2), (7) and (18), one can get

$$
Y_{n}\left(h_{\kappa}^{2} ; I_{\kappa}^{\alpha} f ; x\right)=C_{\kappa, \alpha} \int_{\mathbb{S}^{d}} f(z) Y_{n}\left(h_{\kappa}^{2} ; V_{\kappa}\left(|\langle\cdot, z\rangle|^{\alpha-1}\right) ; x\right) h_{\kappa}^{2}(z) d \omega_{d}(z),
$$

and then applying (21), $Y_{n}\left(h_{\kappa}^{2} ; I_{\kappa}^{\alpha} f ; x\right)=b_{n, \alpha} Y_{n}\left(h_{\kappa}^{2} ; f ; x\right)$ with $b_{n, \alpha}=c_{\kappa}^{-1} C_{\kappa, \alpha} L_{n}$. It is clear that $b_{n, \alpha}=0$ for odd $n$. When $n$ is even, we use 7.311(2) in [12], part (v) in [8, p. 19], and some properties of the gamma function, to get the stated value of $b_{n, \alpha}$.

It is easy to see that (19) and (20) allow us to extend the family $\left\{I_{\kappa}^{\alpha}: \Re \alpha>0, \alpha \neq 1,3,5, \ldots\right\}$ to a larger one, which leads to the following definition. We put $\Pi=\{\alpha \in \mathbb{C}: \alpha \neq 1,3,5, \ldots\}$.

Definition 1. Let $\alpha \in \Pi$. For $f \in L^{1}\left(\mathbb{S}^{d} ; h_{\kappa}^{2}\right)$, we define $I_{\kappa}^{\alpha} f$ by the following $h$-harmonic expansion

$$
I_{\kappa}^{\alpha} f \sim \sum_{n=0}^{\infty} b_{n, \alpha} Y_{n}\left(h_{\kappa}^{2} ; f ; x\right), \quad x \in \mathbb{S}^{d},
$$

where $b_{n, \alpha}$ is given by $(20)$.

It is clear that $I_{\kappa}^{\alpha} f$ is well defined for $f \in C^{\infty}\left(\mathbb{S}^{d}\right)$. In general, $I_{\kappa}^{\alpha} f$ may be a distribution on $\mathbb{S}^{d}$. Since $\left|b_{n, \alpha}\right| \leq c n^{-\lambda_{\kappa}-\Re \alpha}$, then $I_{\kappa}^{\alpha} f \in L^{2}\left(\mathbb{S}^{d} ; h_{\kappa}^{2}\right)$ when $f \in L^{2}\left(\mathbb{S}^{d} ; h_{\kappa}^{2}\right)$ and $\Re \alpha \geq-\lambda_{\kappa}$. For $\Re \alpha<$ $-\lambda_{\kappa}$ and $m \geq-\lambda_{\kappa}-\Re \alpha$, since for $n \geq 1, Y_{n}\left(h_{\kappa}^{2} ; f ; x\right)=\left[n\left(n+2 \lambda_{\kappa}\right)\right]^{-m / 2} Y_{n}\left(h_{\kappa}^{2} ;\left(-\Delta_{h, 0}\right)^{m / 2} f ; x\right)$, we also have $I_{\kappa}^{\alpha} f \in L^{2}\left(\mathbb{S}^{d} ; h_{\kappa}^{2}\right)$ when $f \in W_{m}^{2}\left(\mathbb{S}^{d} ; h_{\kappa}^{2}\right)$. We denote by $W_{m, e}^{2}\left(\mathbb{S}^{d} ; h_{\kappa}^{2}\right)$ the subspace of even functions of $W_{m}^{2}\left(\mathbb{S}^{d} ; h_{\kappa}^{2}\right)$.

Theorem 1. If $\alpha,-2 \lambda_{\kappa}-\alpha \in \Pi$ and $m \geq \max \left\{0,-\lambda_{\kappa}-\Re \alpha\right\}$, then $I_{\kappa}^{\alpha}$ is an isomorphism between $W_{m, e}^{2}\left(\mathbb{S}^{d} ; h_{\kappa}^{2}\right)$ and $W_{m+\lambda_{\kappa}+\Re \alpha, e}^{2}\left(\mathbb{S}^{d} ; h_{\kappa}^{2}\right)$, and

$$
\left(I_{\kappa}^{\alpha}\right)^{-1}=I_{\kappa}^{-2 \lambda_{\kappa}-\alpha} \text {. }
$$

In fact, proceeding the above process, it is not difficult to show that for $f \in W_{m, e}^{2}\left(\mathbb{S}^{d} ; h_{\kappa}^{2}\right)$ with $m \geq \max \left\{0,-\lambda_{\kappa}-\Re \alpha\right\}$, we have $I_{\kappa}^{-2 \lambda_{\kappa}-\alpha} I_{\kappa}^{\alpha} f=f$. For $f \in W_{m^{\prime}, e}^{2}\left(\mathbb{S}^{d} ; h_{\kappa}^{2}\right)$ with $m^{\prime}=m+\lambda_{\kappa}+\Re \alpha$, since $m^{\prime} \geq \max \left\{0,-\lambda_{\kappa}-\Re \alpha^{\prime}\right\}$ with $\alpha^{\prime}=-2 \lambda_{\kappa}-\alpha$, we again have $I_{\kappa}^{-2 \lambda_{\kappa}-\alpha^{\prime}} I_{\kappa}^{\alpha^{\prime}} f=f$, i.e. $I_{\kappa}^{\alpha} I_{\kappa}^{-2 \lambda_{\kappa}-\alpha} f=f$. Combining the two cases proves the theorem.

To go further, for $r \in \mathbb{Z}_{+}$(nonnegative integers) we define

$$
P_{r, \alpha}\left(\Delta_{h, 0}\right)= \begin{cases}\text { the identity operator, } & r=0, \\ 4^{-r} \prod_{j=1}^{r}\left[-\Delta_{h, 0}+a_{j}\right], & r \geq 1,\end{cases}
$$

where $a_{j}=\left(2 \lambda_{\kappa}-2 r+2 j+\alpha-1\right)(2 r-2 j+1-\alpha)$. 
Lemma 1. If $\alpha \in \Pi$, and $r \in \mathbb{Z}_{+}$such that $2 r-2 \lambda_{\kappa}-\alpha \in \Pi$, then for even $n$ and $Y_{n} \in \mathcal{H}_{n}^{h, d+1}$,

$$
P_{r, \alpha}\left(\Delta_{h, 0}\right) I_{\kappa}^{2 r-2 \lambda_{\kappa}-\alpha} I_{\kappa}^{\alpha} Y_{n}=Y_{n}
$$

Proof. From (19) and (20), we have

$$
I_{\kappa}^{2 r-2 \lambda_{\kappa}-\alpha} I_{\kappa}^{\alpha} Y_{n}=\frac{\Gamma\left(\left(n+2 \lambda_{\kappa}-2 r+\alpha+1\right) / 2\right) \Gamma((n+1-\alpha) / 2)}{\Gamma((n+2 r-\alpha+1) / 2) \Gamma\left(\left(n+2 \lambda_{\kappa}+\alpha+1\right) / 2\right)} Y_{n}
$$

Furthermore, from (4),

$$
P_{r, \alpha}\left(\Delta_{h, 0}\right) Y_{n}=\prod_{j=1}^{r}\left(\frac{n+2 \lambda_{\kappa}+\alpha-1}{2}-r+j\right)\left(\frac{n+1-\alpha}{2}+r-j\right) Y_{n} .
$$

Using the properties of $\Gamma$-functions, the result is obtained.

The following theorem is a direct consequence of the above lemma.

Theorem 2. If $\alpha \in \Pi$, and $r \in \mathbb{Z}_{+}$such that $2 r-2 \lambda_{\kappa}-\alpha \in \Pi$ and $r \geq \lambda_{\kappa}+\Re \alpha / 2$, then for even $f \in C^{\infty}\left(\mathbb{S}^{d}\right)$ and $g=I_{\kappa}^{\alpha} f$, we have the inversion formula

$$
f=P_{r, \alpha}\left(\Delta_{h, 0}\right) I_{\kappa}^{2 r-2 \lambda_{\kappa}-\alpha} g .
$$

Now we turn to the inversion problem of the spherical Radon-Dunkl transform $R_{\kappa}$. From (15), (16), (20) and (22), we see that

$$
R_{\kappa} f=\pi^{-1 / 2} \Gamma\left(\lambda_{\kappa}+1 / 2\right) I_{\kappa}^{0} f .
$$

This consistency can be also seen from the following equalities

$$
\frac{\Gamma\left(\lambda_{\kappa}+1\right) \Gamma(\alpha / 2)}{\sqrt{\pi} \Gamma((1-\alpha)) / 2} I_{\kappa}^{\alpha} f(x)=f *_{\kappa} \phi=\int_{-1}^{1} M_{\tau}^{\kappa} f(x) \phi(\tau) w_{\lambda_{\kappa}}(\tau) d \tau
$$

in view of (13) and (18), where $\phi(t)=|t|^{\alpha-1} \in L^{1}\left([-1,1] ; w_{\lambda_{\kappa}}\right)$. Assume that $f \in W_{m}^{2}\left(\mathbb{S}^{d} ; h_{\kappa}^{2}\right)$ with $m>\lambda_{\kappa}+1$. By Proposition 2, for each $x \in \mathbb{S}^{d}, M_{\tau}^{\kappa} f(x)$ is a continuous function of $\tau \in[-1,1]$. Dividing each part of $(24)$ by $\Gamma(\alpha / 2)$ and taking limit for $\alpha \rightarrow 0+$, we regain the relation (23).

From Theorems 1 and 2, we obtain the inversion formulas for the spherical Radon-Dunkl transform $R_{\kappa}$.

Theorem 3. $R_{\kappa}$ is an isomorphism between $W_{m, e}^{2}\left(\mathbb{S}^{d} ; h_{\kappa}^{2}\right)$ and $W_{m+\lambda_{\kappa}, e}^{2}\left(\mathbb{S}^{d} ; h_{\kappa}^{2}\right)$ with $m \geq 0$, and

$$
R_{\kappa}^{-1}=\frac{\sqrt{\pi}}{\Gamma\left(\lambda_{\kappa}+1 / 2\right)} I_{\kappa}^{-2 \lambda_{\kappa}}
$$

Theorem 4. If $r \in \mathbb{Z}_{+}$such that $2 r-2 \lambda_{\kappa} \in \Pi$ and $r \geq \lambda_{\kappa}$, then for even $f \in C^{\infty}\left(\mathbb{S}^{d}\right)$ and $g=R_{\kappa} f$, we have the inversion formula

$$
f=\frac{\sqrt{\pi}}{\Gamma\left(\lambda_{\kappa}+1 / 2\right)} P_{r, 0}\left(\Delta_{h, 0}\right) I_{\kappa}^{2 r-2 \lambda_{\kappa}} g
$$

For a special case, we have some simple inversion formulas for $R_{\kappa}$, which are interesting generalizations of those about the usual spherical Radon transform (see $[15,16,22]$ ). 
Corollary 2. If $\lambda_{\kappa}$ is a positive integer, then an even $f \in C^{\infty}\left(\mathbb{S}^{d}\right)$ can be recovered by

(i) $f=c^{\prime} P_{r, 0}\left(\Delta_{h, 0}\right) R_{\kappa} R_{\kappa} f$,

with $r=\lambda_{\kappa}, c^{\prime}=\pi / \Gamma\left(\lambda_{\kappa}+1 / 2\right)^{2}$, and

$$
P_{r, 0}\left(\Delta_{h, 0}\right)=4^{-r} \prod_{j=1}^{r}\left[-\Delta_{h, 0}+(2 j-1)(2 r-2 j+1)\right] ;
$$

and

(ii) $f=c^{\prime \prime} P_{r, 0}\left(\Delta_{h, 0}\right)\left[\int_{\mathbb{S}^{d}} R_{\kappa} f(y) V_{\kappa}(|\langle x, \cdot\rangle|)(y) h_{\kappa}^{2}(y) d \omega_{d}(y)\right]$,

with $r=\lambda_{\kappa}+1, c^{\prime \prime}=-2 \pi^{3 / 2} c_{\kappa} /\left[\Gamma\left(\lambda_{\kappa}+1\right) \Gamma\left(\lambda_{\kappa}+1 / 2\right)^{2}\right]$, and

$$
P_{r, 0}\left(\Delta_{h, 0}\right)=4^{-r} \prod_{j=1}^{r}\left[-\Delta_{h, 0}+(2 j-3)(2 r-2 j+1)\right] .
$$

\section{Inversion formulas for $R_{\kappa}$ by means of associated wavelets}

In this section, we shall use, for a suitably chosen $\psi$ defined on $[0, \infty)$, the wavelet-like transform

$$
W_{\kappa} f(t, x)=f *_{\kappa} \psi_{t}(x), \quad \psi_{t}(\tau)=t^{-1} \psi(\tau / t),
$$

for $(t, x) \in(0, \infty) \times \mathbb{S}^{d}$, to present the inverse of the spherical Radon-Dunkl transform $R_{\kappa}$ and itself. Although $R_{\kappa}$ is defined implicity and the intertwining operator $V_{\kappa}$ is involved in the definition of $W_{\kappa}$, the approaches in studying the usual spherical Radon transform (see [21], for example) could be transplanted to $R_{\kappa}$.

The first lemma below reveals a relation of the spherical Radon-Dunkl transform $R_{\kappa}$ with the one-dimensional fractional integral, and the second gives a representation of the successive action of $R_{\kappa}$ and $W_{\kappa}$ to a function. We shall use a modified notation of the fractional integral as

$$
B_{\delta} \phi(u)=\frac{2}{\Gamma(\delta)} \int_{0}^{\sqrt{u}} \phi(v)\left(u-v^{2}\right)^{\delta-1} d v, \quad u>0,
$$

for $\delta>0$, which will simplify some expressions.

Lemma 2. For even function $f \in L^{1}\left(\mathbb{S}^{d} ; h_{\kappa}^{2}\right)$ and $0<s<1$, we have

$$
M_{s}^{\kappa}\left(R_{\kappa} f\right)=\frac{\lambda_{\kappa} \pi^{-1}}{w_{\lambda_{\kappa}}(s)} B_{\lambda_{\kappa}}\left(M_{\tau}^{\kappa} f\right)\left(1-s^{2}\right),
$$

where the action of $B_{\lambda_{\kappa}}$ to $M_{\tau}^{\kappa} f$ is associated with $\tau$-variable.

Proof. From the product formula of the Gegenbauer polynomial $C_{2 n}^{\lambda_{\kappa}}$ (see [8, p. 203]), we have

$$
\frac{C_{2 n}^{\lambda_{\kappa}}(s)}{C_{2 n}^{\lambda_{\kappa}}(1)} \frac{C_{2 n}^{\lambda_{\kappa}}(0)}{C_{2 n}^{\lambda_{\kappa}}(1)}=2 \int_{0}^{1} \frac{C_{2 n}^{\lambda_{\kappa}}\left(u \sqrt{1-s^{2}}\right)}{C_{2 n}^{\lambda_{\kappa}}(1)} w_{\lambda_{\kappa}-1 / 2}(u) d u .
$$

By Proposition $1(i i i)$, the three quotients above are the coefficients of a member $Y_{2 n}$ in $\mathcal{H}_{2 n}^{h, d+1}$ under action of $M_{s}^{\kappa}, R_{\kappa}\left(=M_{0}^{\kappa}\right)$, and $M_{u \sqrt{1-s^{2}}}^{\kappa}$, respectively. Therefore,

$$
M_{s}^{\kappa}\left(R_{\kappa} Y_{2 n}\right)=2 \int_{0}^{1}\left(M_{u \sqrt{1-s^{2}}}^{\kappa} Y_{2 n}\right) w_{\lambda_{\kappa}-1 / 2}(u) d u .
$$

Making substitution of variables $u=v / \sqrt{1-s^{2}},(27)$ is proved for $Y_{2 n}$. By Proposition 1 (ii), both sides of (27) are bounded operators in $L^{1}\left(\mathbb{S}^{d} ; h_{\kappa}^{2}\right)$, and hence, the validity of (27) for general even $f \in L^{1}\left(\mathbb{S}^{d} ; h_{\kappa}^{2}\right)$ follows from density of the set of $h$-harmonics. 
Lemma 3. For even $f \in L^{1}\left(\mathbb{S}^{d} ; h_{\kappa}^{2}\right)$ and $\psi \in L^{1}([0, \infty) ; d x)$, we have

$$
W_{\kappa}\left(R_{\kappa} f\right)(t, x)=\frac{2 \lambda_{\kappa}}{\pi} \int_{0}^{1} M_{s}^{\kappa} f(x)\left(B_{\lambda_{\kappa}} \psi_{t}\right)\left(1-s^{2}\right) d s,
$$

provided the integral on the right-hand side exists with $|f|$ and $|\psi|$ instead of $f, \psi$.

Proof. From (13), (25) and (27), we have

$$
W_{\kappa}\left(R_{\kappa} f\right)(t, x)=\int_{-1}^{1} M_{s}^{\kappa}\left(R_{\kappa} f\right)(x) \cdot \psi_{t}(s) w_{\lambda_{\kappa}}(s) d s=\frac{2 \lambda_{\kappa}}{\pi} \int_{0}^{1} B_{\lambda_{\kappa}}\left(M_{\tau}^{\kappa} f\right)\left(1-s^{2}\right) \cdot \psi_{t}(s) d s,
$$

and then, substituting the formula for $B_{\lambda_{\kappa}}\left(M_{\tau}^{\kappa} f\right)$ from (26), and making changes of variables, we prove the equality in (28).

\section{Theorem 5. Let}

$$
\begin{aligned}
& \int_{0}^{\infty} s^{j} \psi(s) d s=0 \quad \text { for all } \quad j=0,2,4, \ldots, 2\left[\lambda_{\kappa}\right], \\
& \int_{1}^{\infty} s^{\beta}|\psi(s)| d s<\infty \quad \text { for some } \quad \beta>2 \lambda_{\kappa} .
\end{aligned}
$$

Then for even $f \in L^{p}\left(\mathbb{S}^{d} ; h_{\kappa}^{2}\right)(1 \leq p<\infty)$, or $C\left(\mathbb{S}^{d}\right)(p=\infty)$, we have

$$
\lim _{\epsilon \rightarrow 0+}\left\|T_{\epsilon} f-f\right\|_{\kappa, p}=0,
$$

where

$$
T_{\epsilon} f(x)=\tilde{C}_{\psi}^{-1} \int_{\epsilon}^{\infty} \frac{\left(W_{\kappa} g\right)(t, x)}{t^{2 \lambda_{\kappa}+1}} d t, \quad \epsilon>0,
$$

with $g=R_{\kappa} f$ and

$$
\tilde{C}_{\psi}= \begin{cases}-\frac{2 \Gamma\left(1-\lambda_{\kappa}\right)}{\pi} \int_{0}^{\infty} s^{2 \lambda_{\kappa}} \psi(s) d s, & \text { if } \lambda_{\kappa} \bar{\in}, \\ \frac{4(-1)^{\lambda_{\kappa}+1}}{\pi \Gamma\left(\lambda_{\kappa}\right)} \int_{0}^{\infty} s^{2 \lambda_{\kappa}} \psi(s) \log s d s, & \text { if } \lambda_{\kappa} \in \mathbb{N} .\end{cases}
$$

In addition, $\lim _{\epsilon \rightarrow 0+} T_{\epsilon} f(x)=f(x)$ for almost all $x \in \mathbb{S}^{d}$.

Proof. Under the assumptions, by [20, Lemma 4.12], we have $\int_{0}^{\infty}\left|B_{\lambda_{\kappa}} \psi(s)\right| d s<\infty$. To prove (31) in general, we only need to show that it is valid for $Y_{2 n} \in \mathcal{H}_{2 n}^{h, d+1}$, and

$$
\left\|T_{\epsilon} f\right\|_{\kappa, p} \leq c\|f\|_{\kappa, p}, \quad \epsilon>0
$$

where the constant $c$ is independent of $\epsilon$. The key step is to rewrite $T_{\epsilon}$ into a convolution operator with an approximate identity, that is,

$$
T_{\epsilon} f(x)=\frac{2 \lambda_{\kappa}\left(\lambda_{\kappa}+1\right)}{\pi(2 \lambda+1) \tilde{C}_{\psi}} f *_{\kappa} K_{\epsilon}
$$

where

$$
K_{\epsilon}(\tau)=\left[w_{\lambda_{\kappa}+1}(\tau)\right]^{-1}\left(B_{\lambda_{\kappa}+1} \psi\right)\left(\epsilon^{-2}\left(1-\tau^{2}\right)\right) .
$$


Indeed, applying Lemma 3 to (32) gives that

$$
\tilde{C}_{\psi} T_{\epsilon} f(x)=\frac{2 \lambda_{\kappa}}{\pi} \int_{0}^{1} M_{s}^{\kappa} f(x) \tilde{K}_{\epsilon}(s) d s
$$

where $\tilde{K}_{\epsilon}(s)=\int_{\epsilon}^{\infty} \frac{\left(B_{\lambda_{\kappa}} \psi_{t}\right)\left(1-s^{2}\right)}{t^{2 \lambda_{\kappa}+1}} d t$. Inserting the formula of $B_{\lambda_{\kappa}} \psi_{t}$ from (26), and then, making changes of variables by $t=\xi^{-1 / 2}$ and $v=\eta \xi^{-1 / 2}$, we get

$$
\tilde{K}_{\epsilon}(s)=\frac{1}{\Gamma\left(\lambda_{\kappa}\right)} \int_{0}^{\epsilon^{-2}} \int_{0}^{\sqrt{\xi\left(1-s^{2}\right)}} \psi(\eta)\left[\xi\left(1-s^{2}\right)-\eta^{2}\right]^{\lambda_{\kappa}-1} d \eta d \xi .
$$

Changing order of the integrals, it follows that $\tilde{K}_{\epsilon}(s)=\frac{\lambda_{\kappa}+1}{2 \lambda_{\kappa}+1} K_{\epsilon}(s) w_{\lambda_{\kappa}}(s)$. Substituting this into (37) and using (13) yields (35).

By Lemma 2.4 in [21], we have $\int_{0}^{\infty} u^{-1}\left(B_{\lambda_{\kappa}+1} \psi\right)(u) d u=\pi \tilde{C}_{\psi} / \lambda_{\kappa}$, and $B_{\lambda_{\kappa}+1} \psi(u)=O\left(u^{\lambda_{\kappa}}\right)$ for $0<u \leq 1$, and $O\left(u^{-\rho}\right)$ for $u>1$ with some $\rho>0$. From these and in view of (9) and (36), it follows that

$$
\begin{aligned}
L_{2 n}\left(K_{\epsilon}\right) & =\frac{2 \lambda_{\kappa}+1}{\lambda_{\kappa}+1} \int_{0}^{1}\left(B_{\lambda_{\kappa}+1} \psi\right)\left(\frac{1-s^{2}}{\epsilon^{2}}\right) \frac{C_{2 n}^{\lambda_{\kappa}}(s) d s}{\left(1-s^{2}\right) C_{2 n}^{\lambda_{\kappa}}(1)} \\
& =\frac{\lambda_{\kappa}+1 / 2}{\lambda_{\kappa}+1} \int_{0}^{\epsilon^{-2}} u^{-1}\left(B_{\lambda_{\kappa}+1} \psi\right)(u) \frac{C_{2 n}^{\lambda_{\kappa}}\left(\sqrt{1-\epsilon^{2} u}\right)}{\sqrt{1-\epsilon^{2} u} C_{2 n}^{\lambda_{\kappa}}(1)} d u,
\end{aligned}
$$

which approaches to $\frac{\pi\left(\lambda_{\kappa}+1 / 2\right)}{\lambda_{\kappa}\left(\lambda_{\kappa}+1\right)} \tilde{C}_{\psi}$ as $\epsilon \rightarrow+0$. For $Y_{2 n} \in \mathcal{H}_{2 n}^{h, d+1}$, from (8) we have $Y_{2 n} *_{\kappa} K_{\epsilon}=$ $L_{2 n}\left(K_{\epsilon}\right) Y_{2 n}$, and by $(35), \lim _{\epsilon \rightarrow 0+} T_{\epsilon} Y_{n}=Y_{n}$ uniformly on $\mathbb{S}^{d}$.

To prove (34), by (11), it suffices to show $\left\|K_{\epsilon}\right\|_{\lambda_{\kappa}, 1} \leq c$ uniformly for $\epsilon>0$ (essentially for $0<\epsilon \leq 1)$. In fact, similarly to (38), we have $\left\|K_{\epsilon}\right\|_{\lambda_{\kappa}, 1}=L_{0}\left(\left|K_{\epsilon}\right|\right)$, approaching to

$$
\frac{\lambda_{\kappa}+1 / 2}{\lambda_{\kappa}+1} \int_{0}^{\infty} u^{-1}\left|\left(B_{\lambda_{\kappa}+1} \psi\right)(u)\right| d u<+\infty
$$

as $\epsilon \rightarrow+0$. Thus (34), and so (31), are proved.

In order to prove $T_{\epsilon} f$ to be convergent almost everywhere, we need the associated maximal function $T_{*} f(x)=\sup _{0<\epsilon \leq 1}\left|T_{\epsilon} f(x)\right|$. We shall show that $T_{*} f$ is dominated by the maximal function introduced in [35]

$$
\mathcal{M}_{\kappa} f(x)=\sup _{0<\theta \leq \pi} \frac{\int_{0}^{\theta}\left(M_{\cos \varphi}^{\kappa}|f|\right)(x)(\sin \varphi)^{2 \lambda_{\kappa}} d \varphi}{\int_{0}^{\theta}(\sin \varphi)^{2 \lambda_{\kappa}} d \varphi},
$$

for $f \in L^{1}\left(\mathbb{S}^{d} ; h_{\kappa}^{2}\right)$, that is

$$
T_{*} f(x) \leq c \mathcal{M}_{\kappa} f(x), \quad x \in \mathbb{S}^{d} .
$$

The pointwise estimates of $B_{\lambda_{\kappa}+1} \psi(u)$ can be written as $B_{\lambda_{\kappa}+1} \psi(u)=O\left(u^{\lambda_{\kappa}}(u+1)^{-\lambda_{\kappa}-\rho}\right)$, which implies the following estimate for $K_{\epsilon}(\cos \theta)$

$$
K_{\epsilon}(\cos \theta)=O\left(m_{\epsilon}(\theta)\right), \quad m_{\epsilon}(\theta)=\frac{\epsilon^{2 \rho}(\sin \theta)^{-1}}{(\epsilon+\sin \theta)^{2 \lambda_{\kappa}+2 \rho}},
$$

with $\rho>0$. The function $m_{\epsilon}(\theta)$ does not suit the process of integration by part in the proof of Theorem 2.6 in [35], since $m(0)=0$. Here we give a proof for the case. 
From (13) and (35),

$$
\left|T_{\epsilon} f(x)\right| \leq c \int_{0}^{\pi / 2}\left(M_{\cos \theta}^{\kappa}|f|\right)(x) m_{\epsilon}(\theta)(\sin \theta)^{2 \lambda_{\kappa}} d \theta
$$

where the evenness of $M_{\tau}^{\kappa} f$ is used. Splitting the interval $[0, \pi / 2]$ into $\bigcup_{j}\left[2^{j} \epsilon, 2^{j+1} \epsilon\right]$, we evaluate each integral $U_{j}=\int_{2^{j} \epsilon}^{2^{j+1} \epsilon}$ separately. For $j \leq 0$, since $\epsilon+\sin \theta \asymp \epsilon$, we have

$$
U_{j} \leq \frac{c 2^{-j}}{\epsilon^{2 \lambda_{\kappa}+1}} \int_{0}^{2^{j+1} \epsilon}\left(M_{\cos \theta}^{\kappa}|f|\right)(x)(\sin \theta)^{2 \lambda_{\kappa}} d \theta \leq c 2^{2 \lambda_{\kappa} j} \mathcal{M}_{\kappa} f(x)
$$

and for $j>0$, since $\epsilon+\sin \theta \asymp \theta$,

$$
U_{j} \leq \frac{c \epsilon^{2 \rho}}{\left(2^{j} \epsilon\right)^{2 \lambda_{\kappa}+2 \rho+1}} \int_{0}^{2^{j+1} \epsilon}\left(M_{\cos \theta}^{\kappa}|f|\right)(x)(\sin \theta)^{2 \lambda_{\kappa}} d \theta \leq c 2^{-2 \rho j} \mathcal{M}_{\kappa} f(x) .
$$

Collecting these estimates into (40) yields (39).

By Theorem 2.1 in [2], $T_{*}$ is of weak $(1,1)$, and strong $(p, p)$ boundedness. Combining with the uniformly convergence of $T_{\epsilon}$ for $h$-harmonics, for general $f \in L^{1}\left(\mathbb{S}^{d} ; h_{\kappa}^{2}\right), T_{\epsilon} f$ converges to $f$ almost everywhere. The proof of Theorem 5 is completed.

In the following, we state two theorems, without proof, which are analogs of Theorems 1.2 and 1.4 in [21]. One is about the reproducing property of the spherical Radon-Dunkl transform $R_{\kappa}$, and the other illustrates the range $R_{\kappa}\left(L^{1}\left(\mathbb{S}^{d} ; h_{\kappa}^{2}\right)\right)$.

Theorem 6. Let

$$
\int_{0}^{\infty} \psi(s) d s=0, \quad \int_{0}^{\infty}|\psi(s) \log s| d s<\infty .
$$

Then for $f \in L^{p}\left(\mathbb{S}^{d} ; h_{\kappa}^{2}\right)(1 \leq p<\infty)$, or $C\left(\mathbb{S}^{d}\right)(p=\infty)$, we have

$$
\lim _{\epsilon \rightarrow 0+}\left\|\tilde{T}_{\epsilon} f-R_{\kappa} f\right\|_{\kappa, p}=0,
$$

where $\tilde{T}_{\epsilon} f(x)=\bar{C}_{\psi}^{-1} \int_{\epsilon}^{\infty} t^{-1}\left(W_{\kappa} f\right)(t, x) d t(\epsilon>0)$, with $\bar{C}_{\psi}=2 c_{\lambda_{\kappa}} \int_{0}^{\infty} \psi(s) \log \frac{1}{s} d s$.

Theorem 7. Let $\psi$ satisfy conditions (29) and (30), $g \in L^{p}\left(\mathbb{S}^{d} ; h_{\kappa}^{2}\right)(1 \leq p<\infty)$, or $C\left(\mathbb{S}^{d}\right)$ $(p=\infty)$, and $\tilde{C}_{\phi} \neq 0$ be the constant in (33). Then the following statements are equivalent:

(i) $g \in R_{\kappa}\left(L^{p}\left(\mathbb{S}^{d} ; h_{\kappa}^{2}\right)\right)$;

(ii) the integrals $S_{\epsilon} g=\int_{\epsilon}^{\infty} t^{-2 \lambda_{\kappa}-1}\left(W_{\kappa} g\right)(t, x) d t$ converge in the $L^{p}\left(\mathbb{S}^{d} ; h_{\kappa}^{2}\right)$-norm.

If $1<p<\infty$, then $(i)$ and $($ ii) are equivalent to

(iii) $\sup _{\epsilon>0}\left\|S_{\epsilon} g\right\|_{\kappa, p}<\infty$.

\section{Acknowledgments}

This work is supported by the National Natural Science Foundation of China (No. 10571122), the Beijing Natural Science Foundation, the Project of Excellent Young Teachers and the Doctoral Programme Foundation of National Education Ministry of China, and the Project of Beijing Education Ministry. 


\section{References}

[1] Campi S., On the reconstruction of a function on a sphere by its integrals over great circles, Boll. Un. Mat. Ital. C (5) 18 (1981), 195-215.

[2] Dai F., Xu Y., Maximal function and multiplier theorem for weighted space on the unit sphere, J. Funct. Anal. 249 (2007), 477-504, math.CA/0703928.

[3] de Jeu M.F.E., The Dunkl transform, Invent. Math. 113 (1993), 147-162.

[4] Dunkl C.F., Reflection groups and orthogonal polynomials on the sphere, Math. Z. 197 (1988), 33-60.

[5] Dunkl C.F., Differential-difference operators associated to reflection groups, Trans. Amer. Math. Soc. 311 (1989), 167-183.

[6] Dunkl C.F., Integral kernels with reflection group invariance, Canad. J. Math. 43 (1991), 1213-1227.

[7] Dunkl C.F., Hankel transforms associated to finite reflection groups, in Hypergeometric Functions on Domains of Positivity, Jack Polynomials and Applications (Tampa, 1991), Contemp. Math. 138 (1992), 123138.

[8] Dunkl C.F., Xu Y., Orthogonal polynomials of several variables, Encyclopedia of Mathematics and its Applications, Vol. 81, Cambridge University Press, Cambridge, 2001.

[9] Erdélyi A., Magnus W., Oberhettinger F., Tricomi F.G., Higher transcendental functions, Vols. I and II, McGraw-Hill Book Company, Inc., New York - Toronto - London, 1953, 1953.

[10] Gindikin S., Reeds J., Shepp L., Spherical tomography and spherical integral geometry, in Tomography, Impedance Imaging, and Integral Geometry (South Hadley, MA, 1993), Lectures in Appl. Math., Vol. 30, Amer. Math. Soc., Providence, RI, 1994, 83-92.

[11] Goodey P., Weil W., Centrally symmetric convex bodies and the spherical Radon transform, J. Differential Geom. 35 (1992), 675-688.

[12] Gradshteyn I.S., Ryzhik L.M., Table of integrals, series, and products, 6th ed., Academic Press, Inc., San Diego, CA, 2000.

[13] Grinberg E.L., Spherical harmonics and integral geometry on projective spaces, Trans. Amer. Math. Soc. 279 (1983), 187-203.

[14] Guillemin V., Radon transform on Zoll surfaces, Adv. in Math. 22 (1976), 85-119.

[15] Helgason S., The Radon transform, 2nd ed., Progress in Mathematics, Vol. 5, Birkhäuser Boston, Inc., Boston, MA, 1999.

[16] Helgason S., Geometric analysis on symmetric spaces, Mathematical Surveys and Monographs, Vol. 39, American Mathematical Society, Providence, RI, 1994.

[17] Kurusa A., The Radon transform on half sphere, Acta Sci. Math. (Szeged) 58 (1993), 143-158.

[18] Rösler M., Positivity of Dunkl's intertwining operator, Duke Math. J. 98 (1999), 445-463, q-alg/9710029.

[19] Rösler M., A positive radial product formula for the Dunkl kernel, Trans. Amer. Math. Soc. 355 (2003), 2413-2438, math.CA/0210137.

[20] Rubin B., Fractional integrals and potentials, Pitman Monographs and Surveys in Pure and Applied Mathematics, Vol. 82, Longman, Harlow, 1996.

[21] Rubin B., Spherical Radon transform and related wavelet transforms, Appl. Comput. Harmon. Anal. 5 (1998), 202-215.

[22] Rubin B., Inversion of fractional integrals related to spherical Radon transform, J. Funct. Anal. 157 (1998), 470-487.

[23] Rubin B., Fractional integrals and wavelet transforms associated with Blaschke-Levy representations on the sphere, Israel J. Math. 114 (1999), 1-27.

[24] Rubin B., Inversion and characterization of the hemiopherical transform, J. Anal. Math. 77 (1999), $105-128$.

[25] Rubin B., Generalized Minkowski-Funk transforms and small denominators on the sphere, Fract. Calc. Appl. Anal. 3 (2000), 177-203.

[26] Rubin B., Inversion formulas for the spherical Radon transform and the generalized cosine transform, Adv. in Appl. Math. 29 (2002), 471-497.

[27] Rubin B., Ryabogin D., The $k$-dimensional Radon transform on the $n$-sphere and related wavelet transforms, in Radon Transforms and Tomography (South Hadley, MA, 2000), Contemp. Math. 278 (2001), 227-239. 
[28] Strichartz R.S., $L^{p}$ estimates for Radon transforms in Euclidean and non-Euclidean spaces, Duke Math. J. 48 (1981), 699-727.

[29] Trimèche K., The Dunkl intertwining operator on spaces of functions and distributions and integral representation of its dual, Integral Transforms Spec. Funct. 12 (2001), 349-374.

[30] Trimèche K., Paley-Wiener theorems for the Dunkl transform and Dunkl translation operators, Integral Transforms Spec. Funct. 13 (2002), 17-38.

[31] Xu Y., Integration of the intertwining operator for $h$-harmonic polynomials associated to reflection groups, Proc. Amer. Math. Soc. 125 (1997), 2963-2973.

[32] Xu Y., Intertwining operator and $h$-harmonic associated with reflection groups, Canad. J. Math. 50 (1998), 193-209.

[33] Xu Y., Approximation by means of $h$-harmonic polynomials on the unit sphere, Adv. Comput. Math. 21 (2004), 37-58.

[34] Xu Y., Weighted approximation of functions on the unit sphere, Constr. Approx. 21 (2005), 1-28, math.CA/0312525.

[35] Xu Y., Almost everywhere convergebce of orthogonal expansions of several variables, Constr. Approx. 22 (2005), 67-93, math.CA/0312526.

[36] Xu Y., Generalized translation operator and approximation in several variables, J. Comput. Appl. Math. 178 (2005), 489-512, math.CA/0401417. 